\title{
Gyrate atrophy of choroid and retina with myopia, cataract and systemic
}

\section{proximal myopathy: A rare case report from rural India}

\author{
Bangal Surekha, Bhandari Akshay, Dhaytadak Priyanka, Gogri Pratik
}

Department Of Ophthalmology, Rural Medical College, Loni.

\section{CASE REPORT}

Bangal S.V. et.al: Gyrate atrophy of choroid and retina with myopia, cataract and systemic proximal myopathy: A rare case report from rural India. AMJ 2012, 5, 12, 639-642. http//dx.doi.org/10.4066/AMJ.2012.1540.

Corresponding Author:

Bangal Surekha V

Department Of Ophthalmology, Rural Medical College

Pravara Institute of Medical Sciences, Loni-413736

Taluka- Rahata, District- Ahmednagar, State- Maharashtra, India.

Email: svb281@rediffmail.com

\section{Abstract}

Gyrate atrophy is a rare metabolic disease with autosomal recessive inheritance pattern characterised by hyperornithinemia and typical ocular findings. This report presents a 17-year-old intellectually challenged girl consulting for a progressive fall of visual acuity with night blindness. Fundus examination showed patches of chorioretinal atrophy with typical scalloped borders and peri vascular pigmentation in the equatorial region. Fundus fluroscein angiography revealed characteristic staining pattern. Other ocular associations included myopia and posterior sub capsular cataract. Progressive systemic proximal myopathy was one of the associated features. Dietary supplementation of vitamin B6 was advised.

Key Words

Chorioretinal gyrate atrophy, myopia, posterior sub capsular cataract, myopathy.

\section{Implications for Practice}

1. What is known about such cases? About 150 biochemically documented cases have been reported in the world literature with about one-third from Finland.

2. What is the key finding reported in this case report? Gyrate atrophy can be diagnosed by ophthalmoscopic findings, Fundus fluroscein angiography (FFA) and by other ancillary investigations.
3. What are the implications for future practice? All patients presenting with gyrate atrophy should be screened for myopia, hyperornithinemia and myopathy. More cases of gyrate atrophy need to be diagnosed and studied from genetic point of view in South East Asians.

\section{Background}

Gyrate atrophy of the choroid and retina was first described by Fuchs in $1896 .{ }^{1}$ It is transmitted as an autosomal recessive trait, which shows deficiency of pyridoxal phosphate-dependent mitochondrial matrix enzyme ornithine aminotransferase (OAT) ${ }^{2}$ and results in a 10 to 20 fold increased level of plasma ornithine. ${ }^{3}$ It is a rare, progressive metabolic choroidal and retinal degeneration. Only 150 biochemically documented cases have been reported to date, one-third being from Finland. ${ }^{4}$ A literature search identified occasional cases from India. ${ }^{5,6}$ The exact mechanism of chorioretinal atrophy in hyperornithinemia is not yet fully understood and a small percentage of the affected people respond to Vitamin B6 supplementation. ${ }^{7}$

\section{Case details}

A 17-year-old female, born out of a non-consanguineous marriage, presented with history of progressive night blindness and visual impairment over four years. She also had difficulty in walking which was gradually progressive for the last eight years and was not associated with visual impairment. Her younger brother had also started getting similar complaints (Figure 7). Ataxic gait with proximal muscle weakness was also noted. On examination there was wasting of proximal limb muscles with muscle power $3 / 5$ in both upper and lower limbs.

Eye examination showed visual impairment in both eyes. Refraction under 2\% Homatropine eye drops, revealed presence of bilateral compound myopic astigmatism. Visual acuity improved from counting fingers at 3 meters to $6 / 18$ in both eyes with - 9.0 D Sph, $-2.0 \mathrm{D} \mathrm{Cyl} \times 90^{\circ}$ in the right eye and -10.0 D Sph, -1.0 D Cyl $\times 140^{\circ}$ in the left eye. Slit lamp bio-microscopic examination of anterior segment showed presence of bilateral early posterior sub capsular cataracts and vitreous degeneration. Ophthalmoscopic examination showed bilateral sharply defined, choroidal atrophic 
patches with scalloped borders typically in the midperipheral zone, resembling gyri of brain (Figure 1).

Atrophic patches showed variable distribution in the form of isolated circular areas to large confluent ones. Myopic changes in the form of large optic disc and temporal crescent with mild retinal arteriolar attenuation were seen (Figure 2). Examination of macula did not reveal any abnormality. Intraocular pressure was normal in both eyes. Axial length of both eyes was $26.93 \mathrm{~mm}$ on ' $A$ ' scan biometry.

Figure 1: Patchy distribution of retinal pigment and choroidal atrophy in the midperiphery

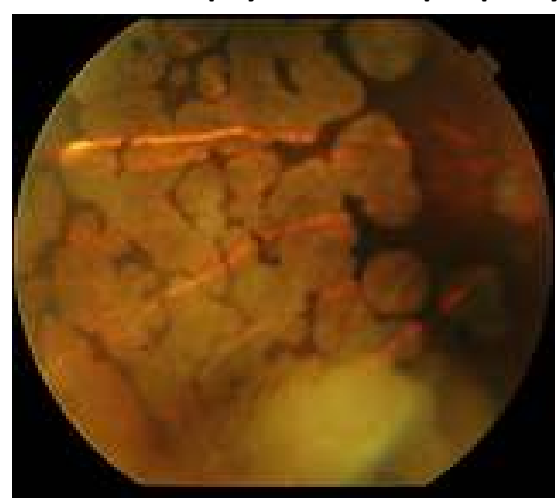

Figure 2: Myopic fundus, temporal crescent

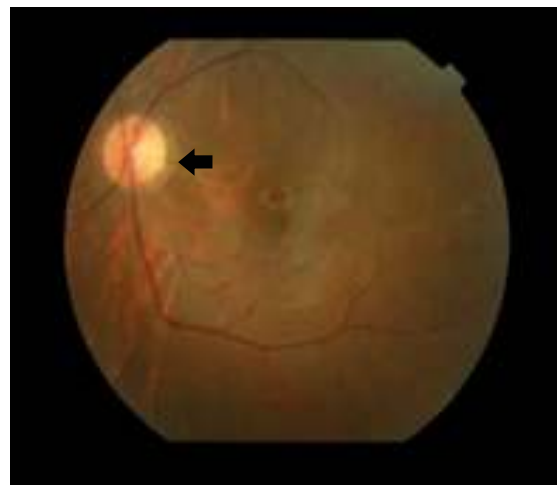

Laboratory investigations were normal except creatinine phosphokinase which was $267.30 \mathrm{IU} / \mathrm{L}$ (normal 0-200 IU/L). Visual fields testing showed concentric loss of field (Figure $5,6)$. Fundus fluroscein angiography showed large choroidal vessels corresponding to ophthalmoscopically visible atrophic areas and the characteristic hyper fluorescence at the borders (Figures 3, 4).
Figure 3: Isolated small gyrate lesions

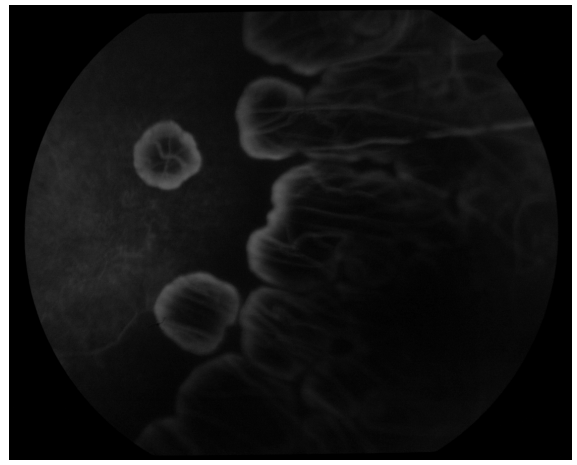

Figure 4: Hyper fluorescent borders of large confluent gyrate lesions with prominent choroidal vasculature

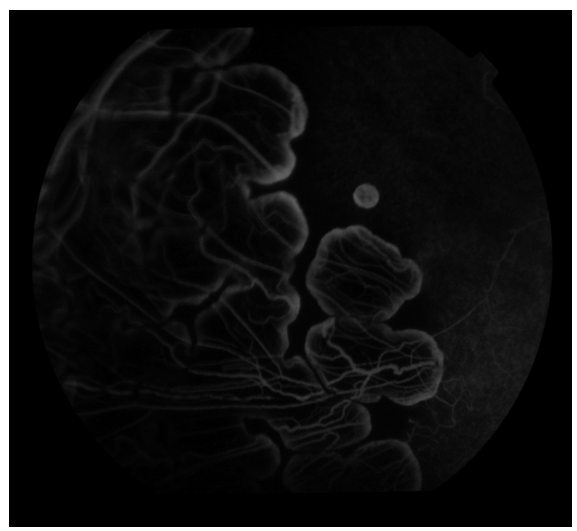

Figure 5: Visual field constriction (Right eye)

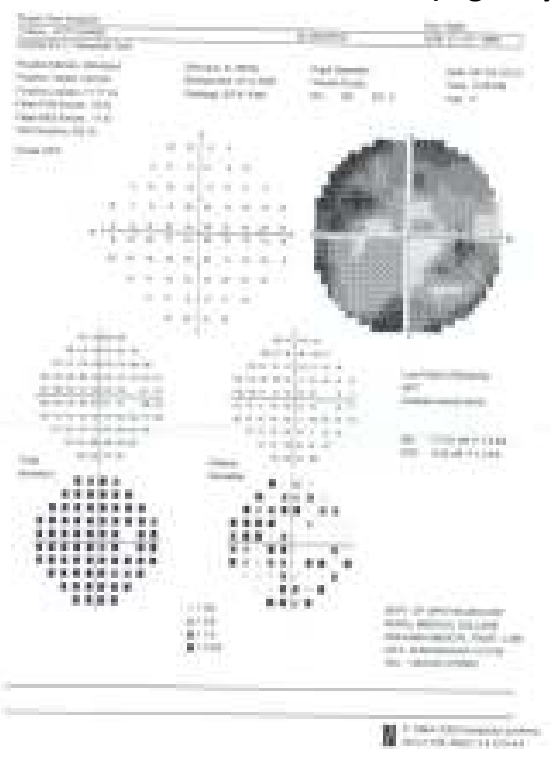


Figure 6: Visual field constriction (Left eye)

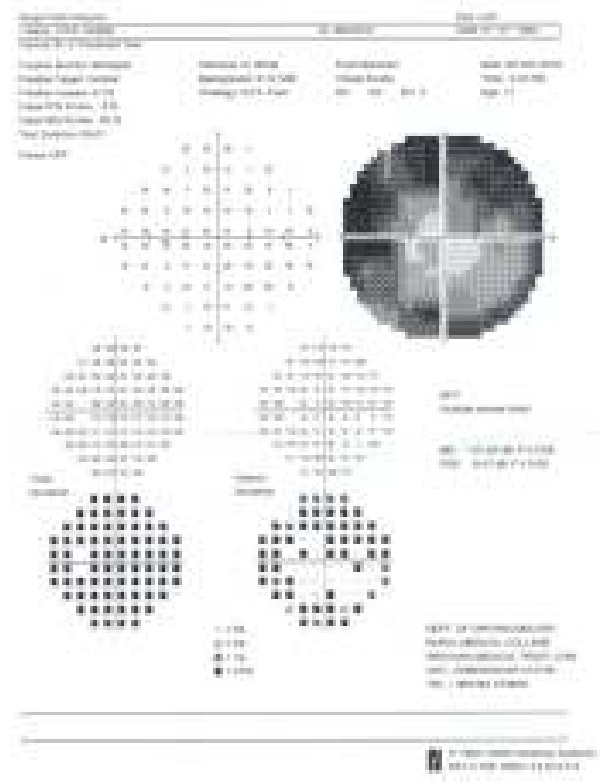

Figure 7: patient and her brother showing muscle wasting

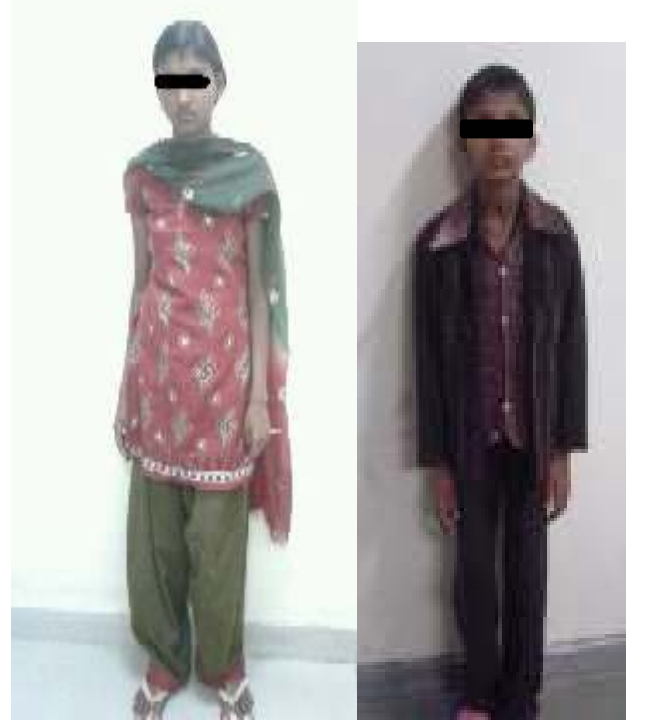

Dietary advice about low protein consumption and daily supplement of Vitamin B6 was emphasised

\section{Discussion}

Gyrate atrophy of the choroid and retina is a rare autosomal recessive dystrophy. In Finland gyrate atrophy occurs in about one in 50,000 individuals. However, the incidence of gyrate atrophy is less than one in 1,000,000 in rest of the world. In India, only two cases have been reported with confirmed biochemical assay. ${ }^{5}$ Amongst reported cases there have been slightly more number of males than females (15:11). Simell and Takki (1973) established the presence of a large increase in plasma ornithine levels with gyrate atrophy. ${ }^{2,3}$ Kupfer, Valle and Valle (1978) identified an absence of ornithine aminotransferase (OAT) as the specific enzyme abnormality associated with gyrate atrophy. $^{8}$

OAT is expressed in most tissues, including kidney, small intestine, liver, and retina. OAT activity is reported to be high in the cell layer external to the photoreceptors in the retinal pigment epithelium. The biochemical mechanism of the chorioretinal degeneration in gyrate atrophy is either the deficiency of essential products or toxicity of the accumulated excessive substrates. The proposed hypotheses for GA are hyperornithinemia, phosphocreatine deficiency, delta 1 pyrrolidine 5 carboxylate/proline deficiency, and excess decarboxylation products (polyamines). ${ }^{9}$

In gyrate atrophy, gradual visual loss usually starts around the age of 10 years and is followed by the appearance of sharply demarcated, circular areas of chorioretinal atrophy with hyper pigmented margins in the mid periphery of the fundus. This appears through the first three decades of life and leads to blindness in the fourth to seventh decades.

Myopia, posterior sub capsular cataracts, and vitreous opacities may also be present. $^{2,3}$ In addition to ocular findings, patients may present with delayed language development and speech defects. Other systemic abnormalities include low intellectual activity, mild proximal muscle weakness, peculiar, sparse scalp hair with areas of alopecia, and bizarre elongated segmented mitochondria in liver biopsies.

Gyrate atrophy of the choroid and retina has been erroneously thought to be a variant of retinitis pigmentosa. Cutler and Fuchs ${ }^{1}$ believed that this disease represented an atypical form of retinitis pigmentosa. This patient also had presence of retinal pigmentation in the equatorial region along with high myopia, posterior sub capsular cataract, vitreous degeneration and characteristic fundus angiographic evidence to establish the diagnosis of gyrate atrophy of choroid (Figures 1, 2). Facilities for the estimation of serum ornithine levels were not available in this rural part of India.

Patients with gyrate atrophy present classically with night blindness between ages 10 to 20 years and are virtually blind between ages 40 to 55 . Improvement in visual acuity and electroretinograms in patients with gyrate atrophy has been reported after a low protein, arginine restricted diet. This has been observed over a 12 month period in a mouse model of OAT deficiency ${ }^{10}$ and had reduced plasma ornithine levels to prevent retinal degeneration. In gyrate 
atrophy, progression of chorioretinal lesions can be slowed if the diet is started at an early age.

Administration of pyridoxine hydrochloride (Vitamin B6) stimulates residual OAT activity and is associated with significant reduction in plasma ornithine levels in some genetically determined responsive patients. ${ }^{7}$ However, treatment requires extensive clinical and biochemical evaluation, regular follow-up and monitoring of patient. In future enzyme replacement and gene therapy may prove to be promising treatment modalities for gyrate atrophy. ${ }^{11}$

\section{References}

1. Fuchs E. Ueberzwei der retinitis pigmentosa verwandtekrankheiten (retinitis punctata albescens und atrophiagyratachoroideae et retinae). Arch Augenheilkd 1896, 32:111-6.

2. Takki K, Simell O. Genetic aspects in gyrate atrophy of the choroid and retina with hyperornithinaemia. $\mathrm{Br}$ J Ophthalmol. 1974 Nov;58(11):907-16.

3. Simell O, Takki K. Raised plasma-ornithine and gyrate atrophy of the choroid and retina. Lancet. 1973 May 12;1(7811):1031-3.

4. Takki KK, Milton RC. The natural history of gyrate atrophy of the choroid and retina. Ophthalmology. 1981 Apr;88(4):292-301..

5. Verma L, Murthy H, Tewari HK, Khosla PK. Gyrate atrophy of choroid and retina. Indian J Ophthalmol. 1989 JulSep;37(3):143-5.

6. Christopher R, Basu SV, Shetty KT. Hyper-ornithinemia associated with Gyrate atrophy of the choroid and the retina: Two cases from India. Ann Clin Biochem. 1999 Jul;36 ( Pt 4):519-22.

7. Javadzadeh A, Gharabaghi D. Gyrate atrophy of the choroid and retina with hyper-ornithinemia responsive to vitamin B6: a case report. J Med Case Rep. 2007 Jun $12 ; 1: 27$.

8. Kaiser-Kupfer M I, Valle D, Valle L A. A specific enzyme defect in Gyrate atrophy. Am J Ophthalmol. 1978 Feb;85(2):200-4.

9. Arshinoff SA, Leung K, Strube YN. Gyrate atrophy. In: William Tasman (ed). Duane's Clinical Ophthalmology. Philadelphia: Lippincott Williams \& Wilkins; 2005.

10. Valle D, Walser M, Brusilow S, Kaiser-Kupfer MI. Gyrate atrophy of the choroid and retina:biochemical considerations and experience with an arginine restricted diet. Ophthalmology. 1981 Apr;88(4):325-30.

11. Weleber RG, Kennaway NG, Buist NR. Gyrate atrophy of the choroid and retina: approaches to therapy. Int Ophthalmol. 1981 Aug;4(1-2):23-32.

\section{ACKNOWLEDGEMENTS}

We express our sincere gratitude to Department of Ophthalmology, Department of Medicine and the staff at the Pravara Rural Hospital for their cooperation and support.

\section{PEER REVIEW}

Not commissioned. Externally peer reviewed.

\section{CONFLICTS OF INTEREST}

$\mathrm{Nil}$

\section{FUNDING}

Nil

\section{PATIENT CONSENT}

The authors declare that

1. They have obtained informed consent for the publication of the details relating to the patient(s) in this report.

2. All possible steps have been taken to safeguard the identity of the patient(s).

3. This submission is compliant with the requirements of local research ethics committees. 